\title{
Natural killer cells inhibit pulmonary metastasis of hepatocellular carcinoma in nude mice
}

\author{
ZAI-FA HONG ${ }^{1,2}$, WEN-XIU ZHAO ${ }^{3}$, ZHEN-YU YIN $^{3}$, CHENG-RONG XIE $^{3}$, YA-PING XU ${ }^{3}$, \\ XIAO-QIN $\mathrm{CHI}^{3}$, ${\text { SHENG } \mathrm{ZHANG}^{3} \text { and XIAO-MIN WANG }}^{3}$ \\ ${ }^{1}$ Department of Hepatobiliary Surgery and Liver Disease Center, \\ Xiamen Hospital of Traditional Chinese Medicine, Xiamen, Fujian 361000; ${ }^{2}$ Post Graduate College, \\ Fujian Medical University, Fuzhou, Fujian 350004; ${ }^{3}$ Department of Hepatobiliary Surgery, \\ Zhongshan Hospital, Xiamen University, Fujian Provincial Key Laboratory of Chronic Liver \\ Disease and Hepatocellular Carcinoma, Xiamen, Fujian 361004, P.R. China
}

Received February 11, 2015; Accepted December 8, 2015

DOI: $10.3892 / \mathrm{ol} .2016 .4170$

\begin{abstract}
Natural killer (NK) cells have been demonstrated to inhibit tumor growth. However, the role of NK cells in the inhibition of hepatocellular carcinoma metastasis is not well understood. The present study aimed to investigate the roles that NK cells may serve in inhibiting hepatocellular carcinoma metastasis. The role of isolated NK cells in the inhibition, proliferation, migration and invasion of the hepatoma cell line, MHCC97-H, was examined in vitro. Additionally, the survival rate of NK cells labeled with carboxyfluorescein diacetate-succinimidyl ester was assessed in vivo. An orthotopic implantation model was used to evaluate the role of NK cells in suppressing MHCC97-H cells in vivo. The effect of interleukin (IL)-2 stimulation on the tumor-inhibitory role of the NK cells was measured indirectly by analyzing the expression of various NK cell receptors and activated NK cell markers. It was observed that the NK cells inhibited the proliferation, migration and invasion of the MHCC97-H cells in vitro. Furthermore, the NK cells demonstrated long-term survival in the livers of the nude mice, and inhibited lung metastasis of hepatocellular carcinoma in vivo. However, liver tumor growth was not inhibited by the NK cells. IL-2 was identified to enhance the tumor-inhibitory effect of NK cells. The present study concludes that IL-2 may enhance the antitumor activity of the NK cells, and thereby inhibit the metastases of hepatocellular carcinoma in mice.
\end{abstract}

Correspondence to: Dr Xiao-Min Wang, Department of Hepatobiliary Surgery, Zhongshan Hospital, Xiamen University, Fujian Provincial Key Laboratory of Chronic Liver Disease and Hepatocellular Carcinoma, 209 South Hubin Road, Xiamen, Fujian 361004, P.R. China

E-mail:wxm@xmzsh.com

Key words: natural killer cells, invasion, hepatocellular carcinoma, pulmonary metastasis, interleukin-2

\section{Introduction}

HCC is one of the most prevalent types of human cancer worldwide, particularly in Southeast Asia (1). The 5-year survival rate of patients with HCC remains poor, with $\sim 600,000$ mortalities each year, despite recent advances in surgical techniques and medical treatment (2). There is a lack of typical symptoms during the early stages of HCC. However, a common clinical manifestation is dull or tingling pain, as well as distension, in the hepatic area, which is the initial symptom in $50 \%$ of HCC patients. Patients may demonstrate additional symptoms, including weakness, weight loss, anorexia, abdominal distension, nausea, vomiting, fever and diarrhea. Hepatomegaly frequently occurs in terminal-stage HCC patients. There are a number of methods via which HCC may be diagnosed, including blood concentration of $\alpha$-fetoprotein, color Doppler ultrasound, $\mathrm{x}$-ray, computed tomography and magnetic resonance imaging (MRI) $(3,4)$. Metastasis is one of the primary causes of the poor prognosis associated with the disease. Tumor metastasis is a difficulty that must frequently be faced when treating hepatocellular carcinoma. Decreased antitumor immunity in patients with cancer has been established to be a major factor associated with the development, progression and metastasis of several types of cancer, including hepatocellular carcinoma (HCC) (5-7). Natural killer (NK) cells serve an important role in the suppression of carcinogenesis (5). Several previous studies have demonstrated that NK cells inhibit tumor growth and that interleukin (IL)-2 is an essential cytokine for NK cell proliferation and activation $(5,8)$. Thus, NK cells may serve as a promising treatment for HCC metastasis. However, the role of NK cells in the inhibition of hepatocellular carcinoma metastasis to the lungs has not been well characterized. In the present study, it was assessed whether NK cells, activated by IL-2, may inhibit the metastasis of hepatocellular carcinoma in vitro and in vivo.

\section{Materials and methods}

Isolation and culture of NK cells. The study protocol conformed to the ethical guidelines of the Declaration of Helsinki and was 
approved by the Institute Research Ethics Committee of the Xiamen Traditional Hospital (Xiamen, China). Blood samples obtained from healthy volunteers were used to separate peripheral blood mononuclear cells (PBMCs). The PBMCs were then used to isolate the NK cells using the Human NK Cell Isolation kit (Miltenyi Biotec $\mathrm{GmbH}$, Bergisch Gladbach, Germany), following the manufacturer's protocols. The NK cells were cultured in RPMI-1640 medium (HyClone; GE Healthcare Life Sciences, Logan, Utah, USA), containing $10 \%$ fetal bovine serum (FBS; Gibco; Thermo Fisher Scientific, Inc., Waltham, MA, USA). Subsequently, the NK cells were activated using recombinant human IL-2 (PeproTech, Inc., Rocky Hill, NJ, USA) at a concentration of $2 \mathrm{pg} / \mathrm{ml}$ (9). The MHCC-97H cells were cultured in RPMI-1640 medium containing $10 \%$ FBS.

Detection of NK cell receptors and activated NK cell markers. Flow cytometry was used to examine the expression of the following cell surface proteins: Cluster of differentiation (CD)56 [phycoerythrin (PE)-labeled mouse anti-human CD56 clone B159; BD Biosciences, San Diego, CA, USA], CD3 [allophycocyanin (APC)-labeled mouse anti-human CD3 clone UCHTI; BD Pharmingen, San Diego, CA, USA], NKG2D [fluorescein isothiocyanate (FITC)-labeled anti-human NKG2D clone 1D11; eBioscience, Inc., San Diego, CA, USA], NKB1 (FITC-labeled anti-human NKB1 clone DX9; BD Pharmingen), perforin (FITC Perforin Reagent set containing FITC-labeled mouse anti-human perforin clone $\delta \mathrm{G} 9$ and FITC-mouse

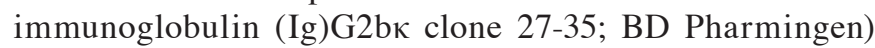
and granzyme (FITC-labeled mouse anti-human granzyme clone GB11; BD Pharmingen). The isotype control included PE-labeled mouse IgG1к (clone MOPC-21), APC-labeled

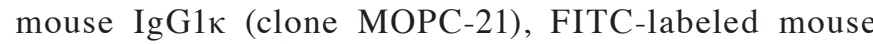

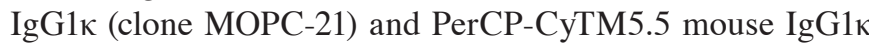
(clone MOPC-21) (all BD Pharmingen). The dilution ratio for all antibodies was 1:100.

Proliferation assay. The NK cells were stimulated with IL-2 $(2 \mathrm{pg} / \mathrm{ml})$ for $48 \mathrm{~h}$ at $37^{\circ} \mathrm{C}$ and were then cocultured with the MHCC97-H cells, which were obtained from the Liver Cancer Institute and Zhongshan Hospital (Shanghai, China). The cells were cultured at a concentration of $2 \times 10^{6}$ cells/plate $\left(1 \times 10^{6}\right.$ cells $\left./ \mathrm{ml}\right)$, using varying amounts of RPMI-1640 medium that contained $10 \%$ FBS. Following a total of $48 \mathrm{~h}$, the culture medium was removed and the MHCC97-H cells were washed with phosphate-buffered saline (PBS). Subsequently, 5-bromo-2'-deoxyuridine (BrdU; Roche Diagnostics, Basel, Switzerland) was added to the MHCC97-H cells, and the cell cultures were reincubated in the RPMI-1640 medium containing $10 \% \mathrm{FBS}$ at $37^{\circ} \mathrm{C}$. Following a total of $4 \mathrm{~h}$, the culture medium was removed and the cells were fixed and permeabilized, and the genomic DNA was denatured in one step through the addition of FixDenat (Roche Diagnostics) according the manufacturer's protocols. The MHCC97-H cells were collected and the BrdU-positive cells were detected with anti-BrdU antibody (PerCP-CyTM5.5-labeled mouse anti-BrdU clone 3D4; 1:100; BD Pharmingen) using flow cytometry.

Wound healing assays. Migration of the NK cells was determined by performing wound healing assays. The MHCC97-H cells were cocultured with the NK cells in 6-well plates (10 ${ }^{6}$ cells/well), with RPMI-1640 medium containing $10 \%$ FBS. Confluent monolayers were wounded in a straight line with a sterile plastic pipette tip, and were subsequently incubated for $72 \mathrm{~h}$ with the NK cells $\left(10^{6}\right.$ cells/well), in $0.2 \%$ FBS medium containing IL-2 (2 pg/ml). In the control group, the monolayers were incubated with medium instead of $\mathrm{NK}$ cells. The NK cell migration was evaluated by comparing the remaining cell-free area with that of the initial wound.

Matrigel invasion assays. Cell invasion was determined using BD BioCoat Matrigel Invasion Chambers (BD Biosciences, Franklin Lakes, NJ, USA). In the NK group, $200 \mu 1$ of $0.2 \%$ FBS medium, containing $5 \times 10^{4}$ MHCC97-H cells and $5 \times 10^{4} \mathrm{NK}$ cells that had been activated with IL-2 (2 pg/ml), were added to the upper compartment of the Transwell chamber, and $600 \mu \mathrm{l}$ of $10 \% \mathrm{FBS}$-containing medium was added to the lower chamber. In the control group, medium was used instead of the $\mathrm{NK}$ cells. Following $48 \mathrm{~h}$ of incubation at $37^{\circ} \mathrm{C}$ in a humidified $\mathrm{CO}_{2}$ incubator, non-invaded cells in the upper chamber were removed with a cotton swab, and the invaded cells were stained and counted using a microscope (x200 magnification) along the longitudinal or transverse axis, including five fields, without any overlap in the fields assessed. The mean value of the cells per field was calculated.

In vivo assessment of $N K$ cell survival. All experimental procedures involving animals were approved by the Animal Care and Use Committee of Xiamen University (Fujian, China). Healthy nude mice were injected through the portal vein with $2 \times 10^{6} \mathrm{NK}$ cells that were stained with carboxyfluorescein diacetate-succinimidyl ester (CFDA-SE; EnoGene Biotech, Co., Ltd., Nanjing, China), a non-fluorescent dye that passively diffuses into the cell cytoplasm. Once inside the cell, the non-fluorescent dye is cleaved by intracellular esterase to become fluorescent and can be detected under a fluorescence microscope. The NK cells were detected 2 weeks after the cell injection using the IX73-U Fluorescence Microscope (Olympus Corporation, Tokyo, Japan).

Tumor growth andmetastasis assays in vivo. The study protocol was approved by the Institutional Animal Care and Use Committee of the Xiamen University (Xiamen, China), and the mice were kept in specific pathogen-free conditions (an environment without specific disease-causing microbe and parasites, including the hepatitis A and E virus; certain non-pathogenic microbes and parasites, including yeasts, were allowed to exist). A hind leg xenograft was established by subcutaneously injecting 2X10 ${ }^{6} \mathrm{MHCC} 97-\mathrm{H}$ cells into 4-week-old nude mice (BALB/c nu/nu: Shanghai SLAC Laboratory Animal Co., Ltd., Shanghai, China). Following a total of 14 days, the subcutaneous tumors were removed and dissected into $1-\mathrm{mm}^{3}$ sections, which were inoculated into the left hepatic lobe of the nude mice to establish orthotopic implantation models. Subsequently, $2 \times 10^{6} \mathrm{NK}$ cells, which had been activated with IL-2, were injected into the orthotopic implantation models through the portal vein, prior to closing of the enterocoelia in the experimental group. In order to continuously activate the NK cells in vivo, IL-2 (1 pg/mg of body weight) was injected into the enterocelia of the orthotopic implantation models from 
A

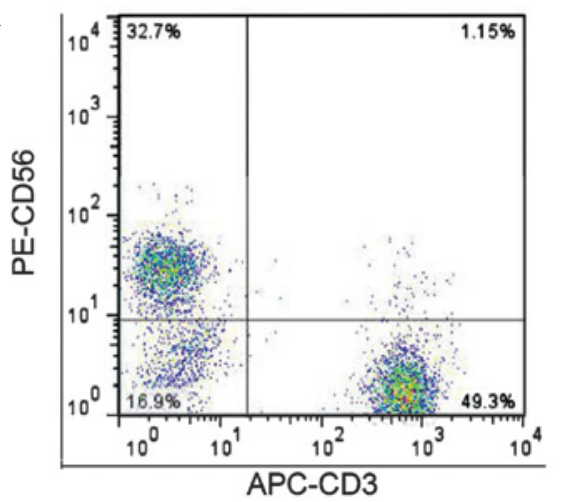

B

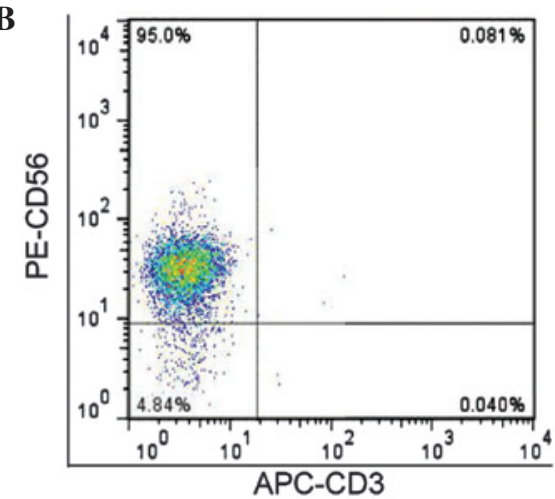

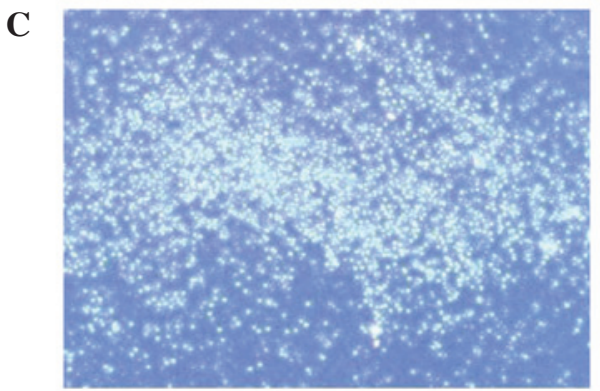

Figure 1. Isolation and culture of the NK cells. (A) CD56 ${ }^{+} \mathrm{CD} 3-$ cells were identified as the marker of the NK cells. The percentage of the NK cells in peripheral blood mononuclear cells was $10-30 \%$. The NK cell percentage of certain samples was $>30 \%$. (B) The purity of the NK cells was $>95 \%$. (C) NK cell culture suspensions formed aggregates or clusters (magnification, x100). NK, natural killer; PE, phycoerythrin; APC, allophycocyanin; CD, cluster of differentiation.

the experimental and control groups on the 5th and 30th days post-surgery. In general, the nude mice exhibited lung metastasis 8 weeks after orthotopic implantation. Tumor growth was monitored, and the nude mice were sacrificed (anesthesia with a $5 \%$ chloral hydrate intraperitoneal injection) on the 56th day post-surgery. The control group was administered PBS instead of NK cells. The mice were sacrificed and the tumors, livers and lungs were removed, weighed, and $5-\mu \mathrm{m}$ sections were fixed in formalin and embedded in paraffin. The liver tumors were detected through MRI, with the tumor volume estimated using the MRI data. The lung metastases were assayed according to methods described by Yang et al (10). The grading system was described as follows: Grade I, <20 tumor cells; grade II, 20-50 tumor cells; grade III, 50-100 tumor cells; and grade IV, $>100$ tumor cells.

Statistical analysis. Data were analyzed using commercially available software (SPSS version 13.0; SPSS, Inc., Chicago, IL, USA). Mean \pm standard deviation values are used to represent replicate experimental data. Student's t-test was used for bivariate analysis, and the rank sum test was used for the assessment of multivariable data. $\mathrm{P}<0.05$ was considered to indicate a statistically significant difference.

\section{Results}

Culture and purity of NK cells. A previous study demonstrated that NK cells isolated from PBMCs express high levels of CD56 and do not express CD3 (5). Therefore, the $\mathrm{CD}^{2} 6^{+} \mathrm{CD} 3^{-}$cells obtained from $\mathrm{PBMCs}$ were considered to be NK cells. The percentage of NK cells in the PBMCs was $10-30 \%$ (Fig. 1A) and the purity of the isolated NK cells was determined to be $>95 \%$ (Fig. 1B). The isolated NK cells exhibited high activity and propagated in clusters in suspension cultures (Fig. 1C).

Role of NK cells in inhibiting MHCC97-H cell mobility in vitro. In order to examine the role of NK cells in the inhibition of hepatocellular carcinoma in vitro, the MHCC97-H cells were cocultured with or without NK cells. The BrdU stain was used to enumerate progenitive MHCC 97-H cells in the presence and absence of the NK cells (Fig. 2A and B). The data demonstrated that the NK cells inhibited the MHCC97-H cell proliferation when the ratio of NK/MHCC97-H was $>0.25 / 1$, and the inhibitory effect peaked at an NK/MHCC97-H ratio of $4 / 1(\mathrm{P}<0.05)$. Therefore, the data indicated that NK cells inhibited the proliferation of the MHCC97-H cells in vitro.

The data also demonstrated that the mobility of the MHCC97-H cells in the wound healing assays was significantly decreased in the presence of the NK cells (Fig. 2C and $\mathrm{D} ; \mathrm{P}<0.05)$. Similarly, the Matrigel invasion assay data indicated that the NK cells significantly decreased migration and invasion of the MHCC97-H cells (Fig. 2E and F; P<0.05).

Role of NK cells in inhibiting MHCC97-H cell survival in vivo. In vivo cell survival experiments demonstrated that NK cells stained with CFDA-SE were localized in the liver tissue. Thus, NK cells may survive in the liver of nude mice (Fig. 3A). To examine the role of NK cells in the inhibition of hepatocellular carcinoma in vivo, the orthotopic implantation models $(n=5)$ were injected with NK cells, and control models $(n=5)$ were injected with PBS. The general state of health, tumor burden in the liver and cancer metastasis to the lungs were examined. 
A
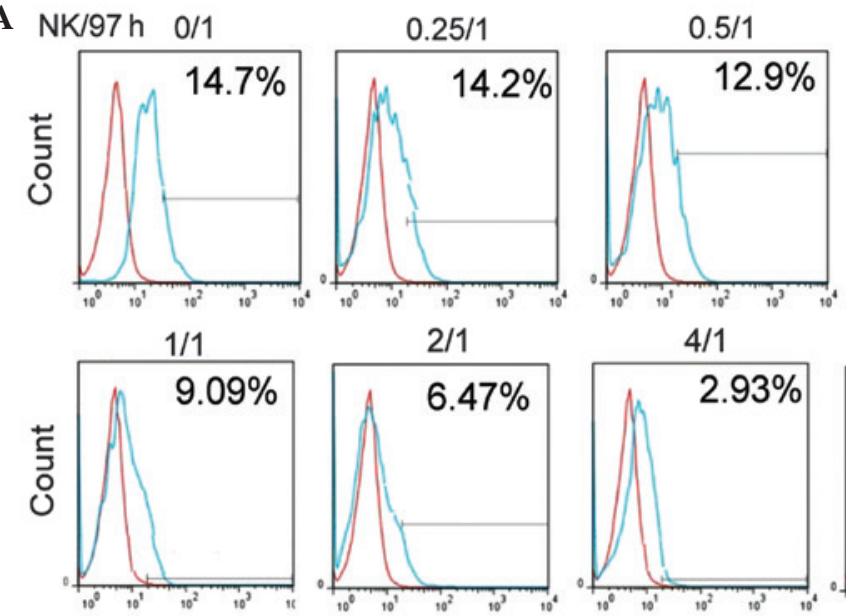

$2 / 1$
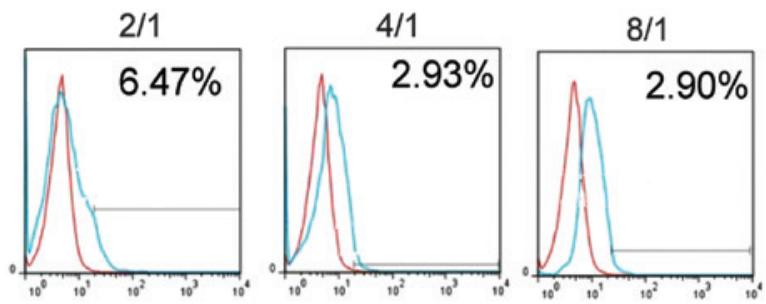

B

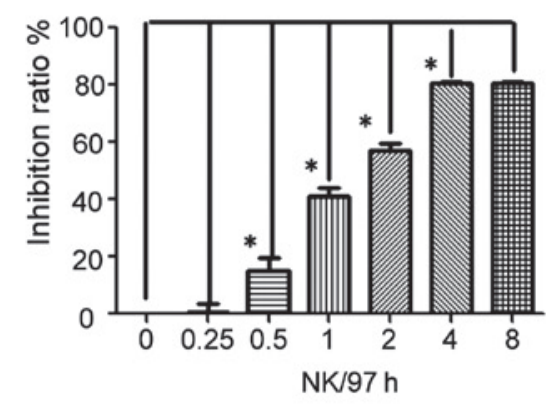

C

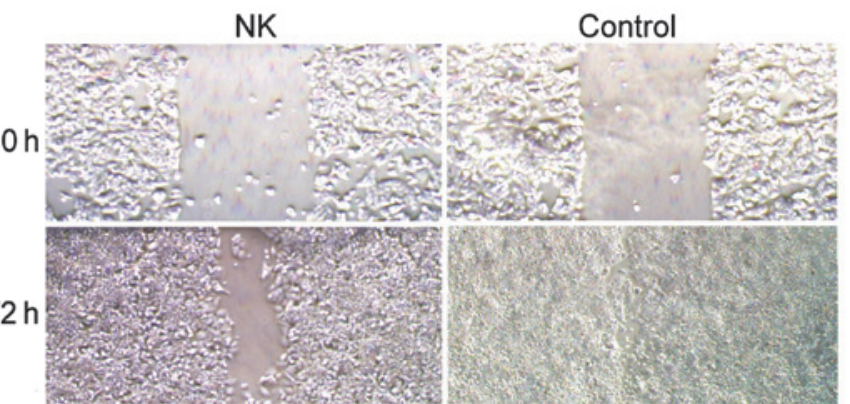

$\mathbf{D}$
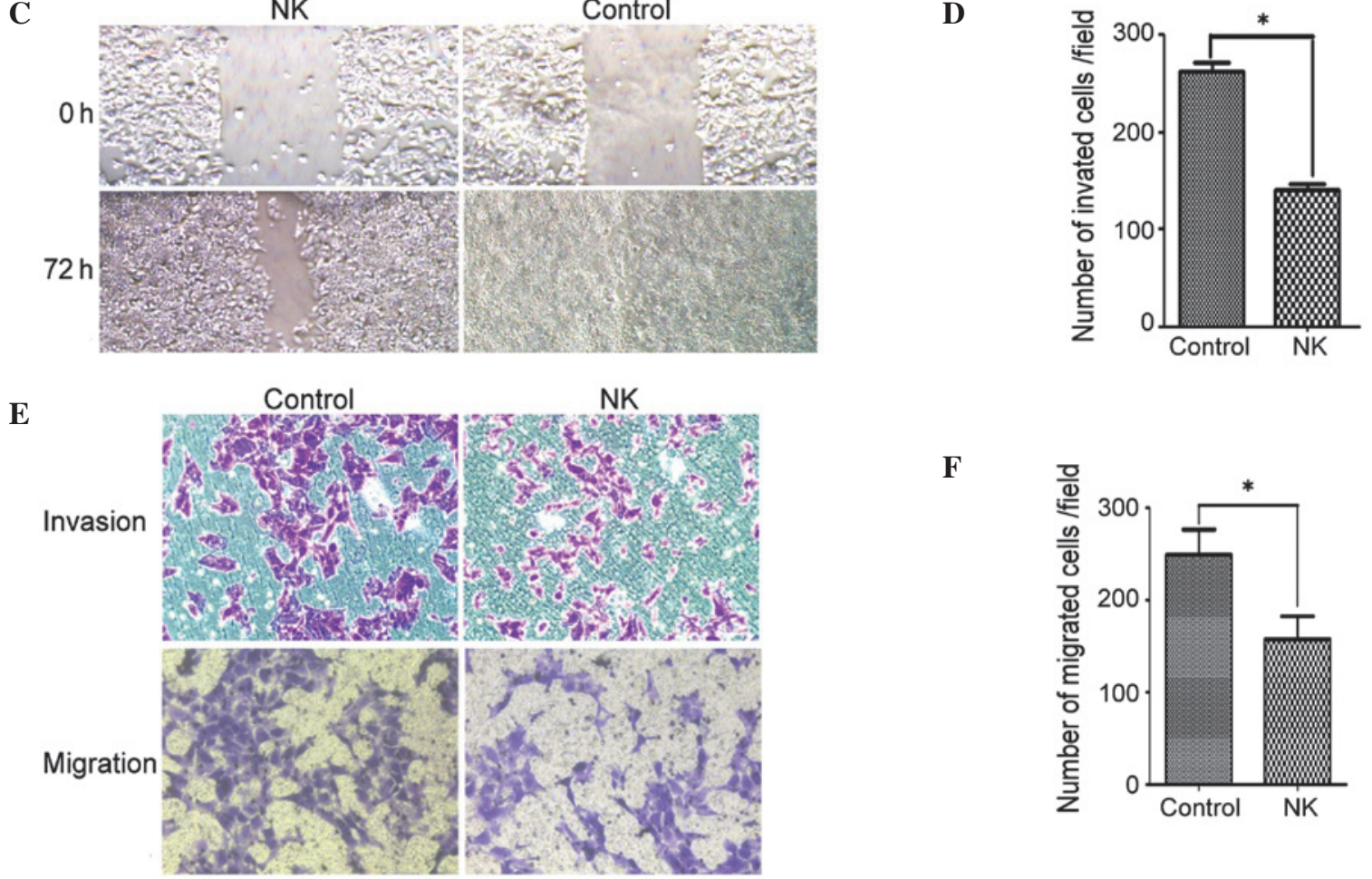

$\mathbf{F}$

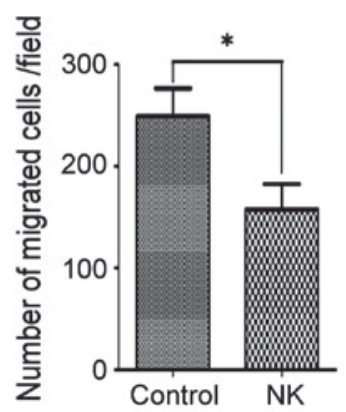

Figure 2. In vitro assessment of the role of NK cells in inhibiting MHCC97-H cell proliferation, migration and invasion. (A) NK cells were cocultured with MHCC97-H cells in different ratios, and the progenitive MHCC97-H cells were detected with anti-BrdU antibody by flow cytometry. The BrdU-positive cells were identified as proliferative cells. (B) Inhibition rate of the indicated cells, calculated as [(the ratio of progenitive cells in the control group - the ratio of progenitive cells in the experimental group) / the ratio of progenitive cells in the control group] x100. (C) Cell-free area of the NK and control groups (magnification, x100). (D) Number of invading cells in the NK and control groups. (E) Histochemical analysis of the NK and control group invasion and migration (staining, crystal violet; magnification, x200). (F) Number of migrating cells in the NK and control groups. Data are presented as the mean \pm standard deviation $(\mathrm{n}=3)$. ${ }^{\mathrm{P}}<0.05$. NK, natural killer; BrdU, 5-bromo-2'-deoxyuridine.

It was observed that the experimental and control animals survived for longer than 6 weeks. The appetite and activity of the two groups were not significantly different.

The volume and weight of the liver tumors of the experimental and control mice were not significantly different (data not shown). The metastatic lesions in the lungs were too small to be detected with MRI (Fig. 3B and C). Metastatic lesions in the lungs of the orthotopic implantation models were measured on the 56th day post-implantation. The total numbers and grades of lung metastatic lesions of the experimental group were considerably lower than those of the control group (Fig. 3D and $\mathrm{E} ; \mathrm{P}<0.05$ ).
Effect of IL-2 stimulation on NK cell function. Previous studies have indicated that IL-2 is required for NK cell growth, proliferation and activation (11). In the present study, NK cells were cultured with or without IL-2 for $48 \mathrm{~h}$ using a previously described protocol (8). The expression of NK group 2, member D (NKG2D; an activating receptor for NK cells) and killer cell immunoglobulin-like receptor, three domains, long cytoplasmic tail, 1 (KIR3DL1; NKB1; an inhibitory receptor) was assessed. Additionally, the expression of perforin and granzyme was also evaluated $(\mathrm{P}<0.05)$. The data indicated that the NK cells exhibited moderate expression levels of NKG2D, perforin and granzyme, and that the expression of the aforementioned 
A

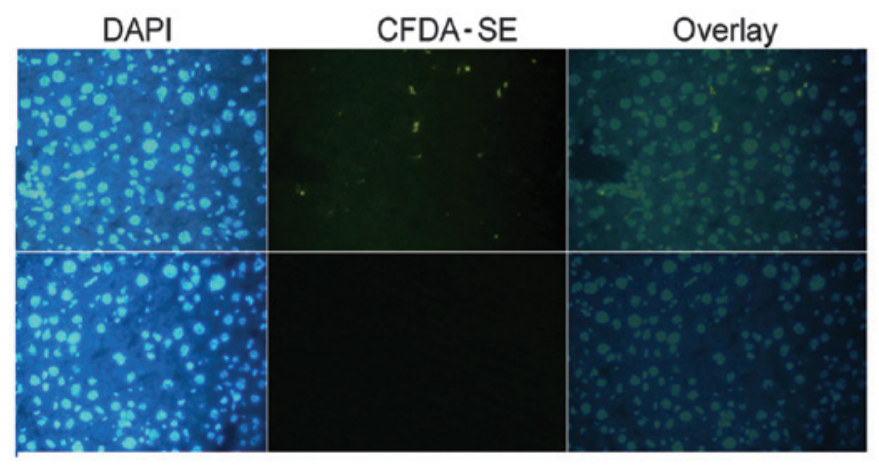

B
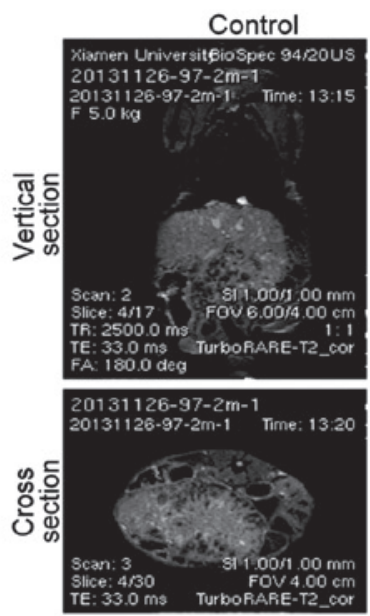

D
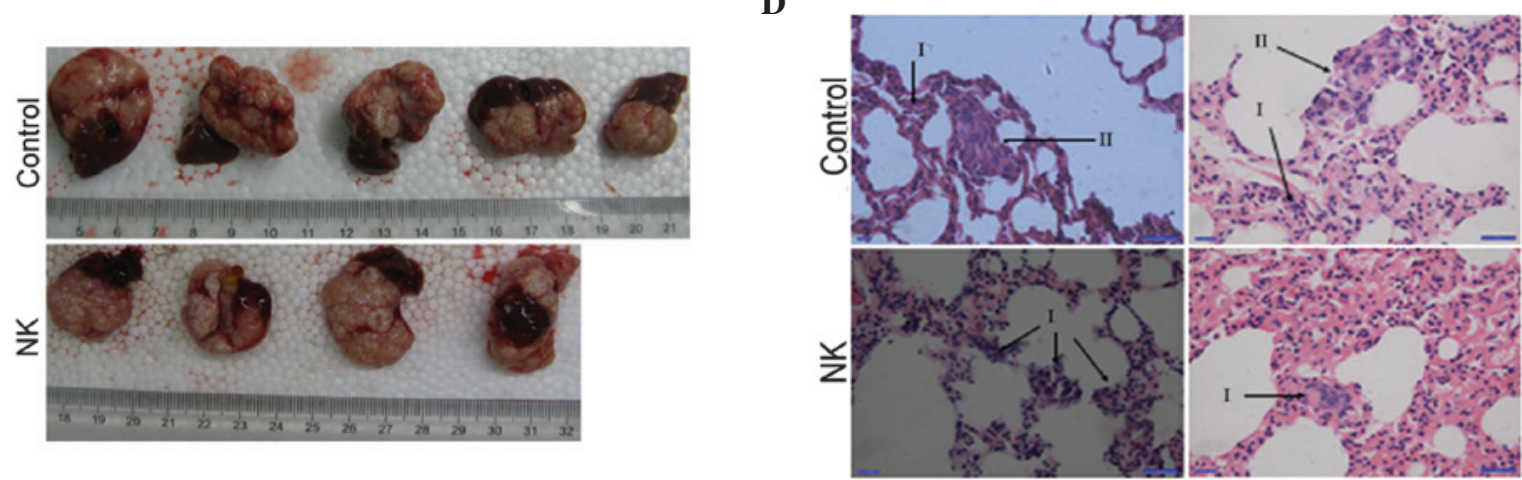

$\mathbf{E}$

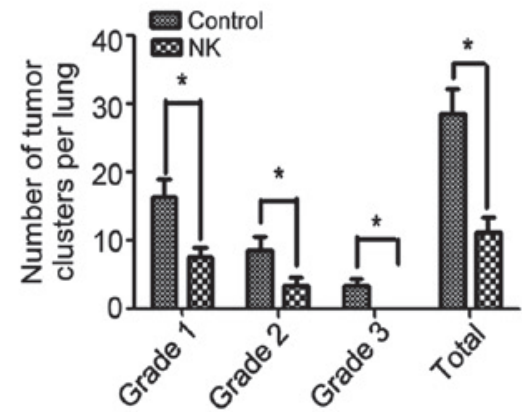

Figure 3. In vivo assessment of the role of NK cells in inhibiting MHCC97-H cell proliferation and invasion. (A) Liver sections were stained with CFDA-SE. The sections were then stained with DAPI dihydrochloride and observed with a fluorescence microscope using blue and green excitation light. Magnification, x200. (B) In the 8th week, the liver tumors were detected using MRI. (C) Tumor volumes of the NK and control groups. (D) Histochemical analysis of the lung metastatic lesions (staining, hematoxylin and eosin; magnification, x200). (E) Number of lung metastatic lesions in the NK and control groups. Data are presented as the mean \pm standard deviation $(n=3)$. $P<0.05$. NK, natural killer; MRI, magnetic resonance imaging; DAPI, 4',6-diamidino-2-phenylindole; CFDA-SE, carboxyfluorescein diacetate succinimidyl ester.

molecules was considerably upregulated following IL-2 stimulation $(\mathrm{P}<0.05)$. However, the NK cells expressed low levels of KIR3DL and the KIR3DL expression was slightly upregulated following IL-2 treatment (Fig. 4; P=0.023).

\section{Discussion}

NK cells are important mediators of the innate immune system, and the number of tumor-infiltrating NK cells may be used as a predictor of liver cancer prognosis (12). A previous study indicated that the function of NK cells is repressed in the liver tumor microenvironment (13). Therefore, strategies to enable NK cell function and activity are required to develop an effective treatment approach.
In the present study, the antitumor activity of NK cells was assessed. The PBMCs separated from blood consisted of $20-30 \% \mathrm{CD}^{2} 6^{+} \mathrm{CD} 3^{-}$cells, which were characterized as the NK cells. The isolated $\mathrm{CD} 56^{+} \mathrm{CD} 3$ cell purity was determined to be $95 \%$. In the presence of IL-2, the NK cells demonstrated robust growth and maintained vitality. In vivo, the NK cells exhibited long-term survival in the liver of the nude mice. This finding was consistent with the findings of previous studies $(8,10)$. NK cell dysfunction in patients with hepatocellular carcinoma has been associated with several factors $(14,15)$, including the suppression of cytokine expression in the tumor environment $(16,17)$. Cytokines, such as IL-12, serve an important role in the activation of NK cells in the tumor microenvironment (18). As aforementioned, IL-2 
A
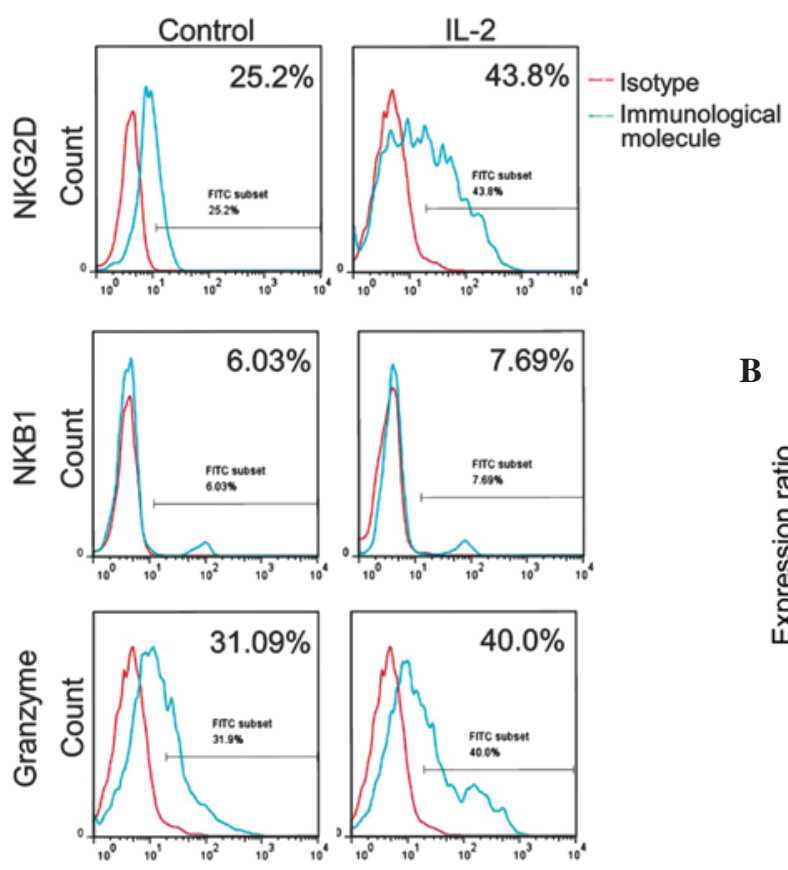

$\mathbf{B}$
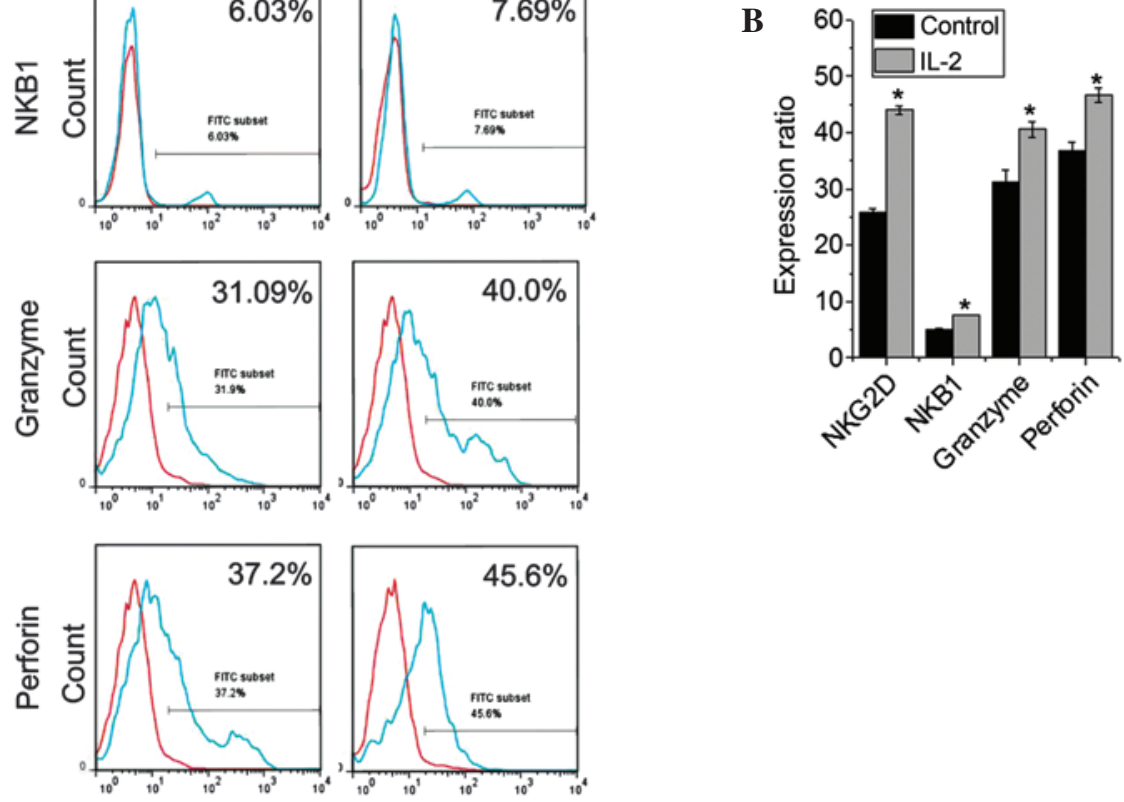

Figure 4. Role of IL-2 in activating NK cells. (A) NK cells were cultured in the presence (IL-2 group) or absence (control group) of IL-2 (2 pg/ml). The immune molecule expression was detected using flow cytometry. (B) The expression of NK cell-associated proteins on the NK cells of the control group and IL-2 group. $\mathrm{NK}$, natural killer; IL, interleukin; FITC, fluorescein isothiocyanate. "P<0.05.

is required for the growth, proliferation and activation of NK cells. Furthermore, NK cells with repressed effector function regain cytolytic function following stimulation with IL-2 (19).

Overall, several studies have demonstrated that NK cells inhibit tumor growth. Maat et al (20) demonstrated that NK cells were involved in the prevention of uveal melanoma metastases. However, few studies have investigated the treatment efficacy of NK cell administration in the prevention of hepatocellular carcinoma metastases. Barkholt et al (8) reported that NK cells, along with IL-2, may be safely administered to patients with hepatocellular carcinoma. In the present in vitro study, the data indicated that IL-2 activated the NK cells, and therefore inhibited MHCC $97-\mathrm{H}$ proliferation, migration and invasion. Furthermore, the in vivo data demonstrated that the NK cells inhibited pulmonary metastases of hepatocellular cancer. However, the tumor burden in the livers obtained from the control and NK groups was not significantly different. The similarity in tumor burden may be due to low levels of tumor necrosis and the poor nutritional intake by the nude mice in the last weeks of the experiments.

The mechanisms underlying the NK-mediated inhibition of tumor invasion and tumor metastasis have not been well characterized. As aforementioned, the cytokines secreted by NK cells may be crucial in activating antitumor immunity. For example, NK cells are a major producer of interferon- $\gamma$, which is critical for the IL-12-induced antitumor effect (21). Furthermore, NK cells may mediate antitumor responses via direct contact with tumor cells or other immune cells. The contact between NK cells and tumor cells may lead to tumor cell immunoediting through cell surface molecular interactions $(22,23)$.

Previous studies have demonstrated that NK cell receptors and their corresponding ligands serve an important role in liver diseases $(24,25)$. NKG2D is an important activating receptor of NK cells, and NKG2D expression has been directly associated with hepatocellular carcinoma prognosis (26). U116 binding protein is an NKG2D ligand and is expressed on tumor cells. The absence of U116 binding protein expression has been identified as an independent factor for recurrent hepatocellular carcinoma (27). NK cell cytotoxicity is mediated through the NKG2D-major histocompatability complex (MHC) class I-related chain A/B interaction (28). In the present study, the expression of NKG2D increased significantly following incubation with IL-2 and the expression of NKB1, an inhibitory receptor, was elevated moderately. The expression of cell markers for activated NK cells (granzyme and perforin) increased significantly. Overall, the data indicate that NK cells exert inhibitory effects on tumor cell proliferation, migration and invasion.

Killer immunoglobulin receptors (KIRs) are NK cell receptors that are associated with liver cancer incidence. KIRs are involved in the regulation of NK cell activation through recognition and binding with specific human leukocyte antigen (HLA) class I allotypes. Thus, the type of HLAs expressed on 
tumor cells may determine the antitumor efficacy of the NK cells (29). NKB1 (KIR3DL1) is a member of the KIR family. The interaction of NKB1 with specific HLA-B antigens on a target cell inhibits cell-mediated cytotoxicity, possibly by delivering a negative signal that prevents NK cell activation. Therefore, the interactions between MHC I and KIRs may regulate NK responses to antigenic challenge (30).

The mechanisms underlying the antitumor and anti-metastatic activity of NK cells have not been well characterized. Few studies have indicated that NK cells may mediate the removal of micro-metastatic cells from circulation and attack metastatic cells present inside organs. Therefore, methods to further improve the antitumor functions of NK cells are of considerable interest. The role of signaling pathways and gene expression in the development of NK cells has been previously investigated. One study demonstrated that activation of the Notch signaling pathway supports the development of cytokine-producing NK cells that are hyporesponsive and have low receptor and poor effector functions (31). Furthermore, microRNA-30c-1 has been identified to strengthen NK cell cytotoxicity against hepatocellular carcinoma cells by regulating the expression of the transcription factor homeobox containing 1 (32). When combined, these studies indicate that the NK cell antitumor activity may be improved by regulating the signal transduction pathways or the gene expression of NK cells.

In conclusion, in the present study, it was demonstrated that NK cells inhibited the proliferation, migration and invasion of the MHCC97-H cells in vitro, and that the NK cells survived in the livers of the nude mice. Furthermore, the NK cells inhibited lung metastasis of hepatocellular carcinoma in vivo. However, the NK cells did not inhibit tumor growth in the liver of the nude mice. The data also indicated that IL-2 may enhance NK cell-mediated metastasis inhibition by inducing the expression of immunologically relevant molecules. However, further investigation is required to identify the mechanisms that enable NK cells to inhibit cancer metastasis from the liver to the lungs.

\section{Acknowledgements}

This study was supported by grants from the National Natural Science Foundation of China (no. 81171976) and the Fujian University of Traditional Chinese Medicine (no. XB2015039).

\section{References}

1. But DY, Lai CL and Yuen MF: Natural history of hepatitis-related hepatocellular carcinoma. World J Gastroenterol 14: 1652-1656, 2008.

2. Hao K, Luk JM, Lee NP, Mao M, Zhang C, Ferguson MD, Lamb J, Dai H, Ng IO, Sham PC and Poon RT: Predicting prognosis in hepatocellular carcinoma after curative surgery with common clinicopathologic parameters. BMC Cancer 9: 389, 2009.

3. Sangiovanni A and Colombo M: Treatment of hepatocellular carcinoma: Beyond international guidelines. Liver Int 36 (Suppl 1): 124-129, 2016.

4. Invernizzi F, Viganò M, Grossi G and Lampertico P: The prognosis and management of inactive HBV carriers. Liver Int 36 (Suppl 1): 100-104, 2016

5. Seki S, Nakashima H, Nakashima M and Kinoshita M: Antitumor immunity produced by the liver Kupffer cells, NK cells, NKT cells, and CD8 CD122 T cells. Clin Dev Immunol 2011: 868345, 2011.

6. Geissler M, Mohr L and Blum HE: Immunotherapy of hepatocellular carcinoma. Dtsch Med Wochenschr 126: 1464-1466, 2001 (In German).
7. Gersuk GM, Westermark B, Mohabeer AJ, Challita PM, Pattamakom S and Pattengale PK: Inhibition of human natural killer cell activity by platelet-derived growth factor (PDGF). III. Membrane binding studies and differential biological effect of recombinant PDGF isoforms. Scand J Immunol 33: 521-532, 1991.

8. Barkholt L, Alici E, Conrad R, Sutlu T, Gilljam M, Stellan B, Christensson B, Guven H, Björkström NK, Söderdahl G et al: Safety analysis of ex vivo-expanded NK and NK-like T cells administered to cancer patients: A phase I clinical study. Immunotherapy 1: 753-764, 2009.

9. Doskali M, Tanaka Y, Ohira M, Ishiyama K, Tashiro H, Chayama K and Ohdan H: Possibility of adoptive immunotherapy with peripheral blood-derived $\mathrm{CD} 3{ }^{-} \mathrm{CD} 56^{+}$and $\mathrm{CD} 3^{+} \mathrm{CD} 56^{+}$cells for inducing antihepatocellular carcinoma and antihepatitis $\mathrm{C}$ virus activity. J Immunother 34: 129-138, 2011.

10. Yang X, Liang L, Zhang XF, Jia HL, Qin Y, Zhu XC, Gao XM, Qiao P, Zheng Y, Sheng YY, et al: MicroRNA-26a suppresses tumor growth and metastasis of human hepatocellular carcinoma by targeting interleukin-6-Stat3 pathway. Hepatology 58: 158-170, 2013.

11. Ciaglia E, Pisanti S, Picardi P, Laezza C, Sosa S, Tubaro A, Vitale M, Gazzerro P, Malfitano AM and Bifulco M: N6-isopentenyladenosine affects cytotoxic activity and cytokines production by IL-2 activated NK cells and exerts topical anti-inflammatory activity in mice. Pharmacol Res 89: $1-10,2014$.

12. Zhu LY, Zhou J, Liu YZ and Pan WD: Prognostic significance of natural killer cell infiltration in hepatocellular carcinoma. Ai Zheng 28: 1198-1202, 2009 (In Chinese).

13. Matsuoka S, Maeda N, Izumiya C, Yamashita C, Nishimori Y and Fukaya T: Expression of inhibitory-motif killer immunoglobulin-like receptor, KIR2DL1, is increased in natural killer cells from women with pelvic endometriosis. Am J Reprod Immunol 53: 249-254, 2005.

14. Li T, Yang Y, Hua X, Wang G, Liu W, Jia C, Tai Y, Zhang Q and Chen G: Hepatocellular carcinoma-associated fibroblasts trigger NK cell dysfunction via PGE2 and IDO. Cancer Lett 318: 154-161, 2012.

15. Ju Y, Hou N, Meng J, Wang X, Zhang X, Zhao D, Liu Y, Zhu F, Zhang L, Sun W, et al: T cell immunoglobulin- and mucin-domain-containing molecule-3 (Tim-3) mediates natural killer cell suppression in chronic hepatitis B.J Hepatol 52: 322-329, 2010.

16. Ohira M, Nishida S, Tryphonopoulos P, Tekin A, Selvaggi G, Moon J, Levi D, Ricordi C, Ishiyama K, Tanaka Y, et al: Clinical-scale isolation of interleukin-2-stimulated liver natural killer cells for treatment of liver transplantation with hepatocellular carcinoma. Cell Transplant 21: 1397-1406, 2012.

17. Tsunematsu H, Tatsumi T, Kohga K, Yamamoto M, Aketa H, Miyagi T, Hosui A, Hiramatsu N, Kanto T, Hayashi N and Takehara T: Fibroblast growth factor-2 enhances NK sensitivity of hepatocellular carcinoma cells. Int J Cancer 130: 356-364, 2012.

18. Mikuriya Y, Tashiro H, Kuroda S, Nambu J, Kobayashi T, Amano H, Tanaka Y and Ohdan H: Fatty liver creates a pro-metastatic microenvironment for hepatocellular carcinoma through activation of hepatic stellate cells. Int J Cancer 136: E3-E13, 2015.

19. Faridi RM, Das V, Tripthi G, Talwar S, Parveen F and Agrawal S: Influence of activating and inhibitory killer immunoglobulin-like receptors on predisposition to recurrent miscarriages. Hum Reprod 24: 1758-1764, 2009.

20. Maat W, van der Slik AR, Verhoeven DH, Alizadeh BZ, Ly LV, Verduijn W, Luyten GP, Mulder A, van Hall T, Koning F, et al: Evidence for natural killer cell-mediated protection from metastasis formation in uveal melanoma patients. Invest Ophthalmol Vis Sci 50: 2888-2895, 2009

21. Uemura A, Takehara T, Miyagi T, Suzuki T, Tatsumi T, Ohkawa K, Kanto T, Hiramatsu N and Hayashi N: Natural killer cell is a major producer of interferon gamma that is critical for the IL-12-induced anti-tumor effect in mice. Cancer Immunol Immunother 59: 453-463, 2010.

22. Wu Y, Kuang DM, Pan WD, Wan YL, Lao XM, Wang D, Li XF and Zheng L: Monocyte/macrophage-elicited natural killer cell dysfunction in hepatocellular carcinoma is mediated by CD48/2B4 interactions. Hepatology 57: 1107-1116, 2013.

23. Yoon JC, Lim JB, Park JH and Lee JM: Cell-to-cell contact with hepatitis $\mathrm{C}$ virus-infected cells reduces functional capacity of natural killer cells. J Virol 85: 12557-12569, 2011.

24. Yamagiwa S, Kamimura $H$ and Ichida T: Natural killer cell receptors and their ligands in liver diseases. Med Mol Morphol 42: 1-8, 2009. 
25. Hoechst B, Voigtlaender T, Ormandy L, Gamrekelashvili J, Zhao F, Wedemeyer H, Lehner F, Manns MP, Greten TF and Korangy F: Myeloid derived suppressor cells inhibit natural killer cells in patients with hepatocellular carcinoma via the NKp30 receptor. Hepatology 50: 799-807, 2009.

26. Konjević G, Mirjacić Martinović K, Vuletić A, Jović V, Jurisić V, Babović N and Spuzić I: Low expression of CD161 and NKG2D activating NK receptor is associated with impaired NK cell cytotoxicity in metastatic melanoma patients. Clin Exp Metastasis 24: $1-11,2007$.

27. Kamimura H, Yamagiwa S, Tsuchiya A, Takamura M, Matsuda Y, Ohkoshi S, Inoue M, Wakai T, Shirai Y, Nomoto M and Aoyagi Y: Reduced NKG2D ligand expression in hepatocellular carcinoma correlates with early recurrence. J Hepatol 56: 381-388, 2012.

28. Morisaki T, Onishi H, Koya N, Kiyota A, Tanaka H, Umebayashi M, Ogino T, Nagamatsu I and Katano M: Combinatorial cytotoxicity of gemcitabine and cytokine-activated killer cells in hepatocellular carcinoma via the NKG2D-MICA/B system. Anticancer Res 31: 2505-2510, 2011.
29. Pan N, Jiang W, Sun H, Miao F, Qiu J, Jin H, Xu J, Shi Q, Xie W and Zhang J: KIR and HLA loci are associated with hepatocellular carcinoma development in patients with hepatitis B virus infection: A case-control study. PLoS One 6: e25682, 2011.

30. Townsley E, O'Connor G, Cosgrove C, Woda M, Co M, Thomas SJ, Kalayanarooj S, Yoon IK, Nisalak A, Srikiatkhachorn A, et al: Interaction of a dengue virus NS1-derived peptide with the inhibitory receptor KIR3DL1 on natural killer cells. Clin Exp Immunol Oct 6, 2015 (Epub ahead of print)

31. Bachanova V, McCullar V, Lenvik T, Wangen R, Peterson KA, Ankarlo DE, Panoskaltsis-Mortari A, Wagner JE and Miller JS: Activated notch supports development of cytokine producing NK cells which are hyporesponsive and fail to acquire NK cell effector functions. Biol Blood Marrow Transplant 15: 183-194, 2009.

32. Gong J, Liu R, Zhuang R, Zhang Y, Fang L, Xu Z, Jin L, Wang T, Song C, Yang K, et al: miR-30c-1* promotes natural killer cell cytotoxicity against human hepatoma cells by targeting the transcription factor HMBOX1. Cancer Sci 103: 645-652, 2012. 